\title{
An Empirical Study of IPV6 Multicast Routing over a Virtual Local Area Network
}

\author{
A. A. Obiniyi \\ Department of Mathematics, \\ Ahmadu Bello University Zaria, Nigeria
}

\author{
Ahiaba Solomon \\ Department of Mathematics, \\ Ahmadu Bello University Zaria, Nigeria
}

\begin{abstract}
The Internet Protocol Version 6 was designed to efficiently improve on the existing functionalities of Internet Protocol Version 4 and to introduce new constructs that it lacks. Though IPv6 is not an extension of IPv4, as the two protocols have different specifications. Both the new and the formal protocol use multicast routing for many of their operations; this implies multicast routing is core in the protocols. This experiment became imperative especially in this era everyone is looking forward to using IPv6 as the default network. This paper tested the performance of IPv6 multicast routing over a virtual local area network. Graphical Network Simulator 3 was used to configure the network and Microsoft Hyper-V was used as the hypervisor on which the six virtual machines (hosts) reside. Parameters such as throughput, latency variations, data loss and the network over heads were examined. The experiment has shown that, IPv6 multicast routing over a virtual network has $100 \%$ throughput, the jitter (variations in latency) varies among the hosts in all the running scenarios, but low and stabled jitters were noticed as the running duration increases and the number of streaming increase from one multicast stream to running two multicast streams simultaneously. There was no data loss.
\end{abstract}

\section{Keywords}

IPv4, IPv6, Unicast routing, Multicast routing, virtual machines, Graphical Network Simulator 3(GNS3), Protocol Independent Multicast-Sparse Mode (PIM-SM),Multicast Listener Discovery (MLD), Microsoft Hyper-V.

\section{INTRODUCTION}

The major changes from IPv4 to IPv6 fall primarily into the following categories; expanded addressing capabilities, header format simplification, improved support for extensions and options, flow labeling capability [2]. The challenges of IPv6 are directly connected to its protocol stack specification (multicast and header specification), [10]. This means multicast routing contributed to challenges facing IPv6 as a result of new it assume which replaces broadcast. Therefore, testing IPv6 multicast routing became very necessary in this era of IPv4 to IPv6 transition.

\subsection{Summary of IPv6}

Internet Protocol Version 6 (IPv6) which is a new version of the Internet Protocol, was specified in [8]. It was first introduced in 1998 by the Internet Engineering Task Force (IETF) in order to replace IPv4.

The new protocol has 2128 addresses compare to 232 addresses of IPv4, it is fast, efficient, more secured and support mobile applications [3].

\subsection{The IPv6 Header}

The standard specification for IPv6 header, according to [8] is displayed in figure 1.1

\begin{tabular}{|c|c|c|c|}
\hline Version & \multicolumn{2}{|c|}{ Traffic class } & Flow Label \\
\hline Playload & igth & Next Header & Hop Limit \\
\hline \multicolumn{4}{|c|}{ Source Address } \\
\hline \multicolumn{4}{|c|}{ Destination Address } \\
\hline
\end{tabular}

Figure 1.1 The IPv6 Header [11]

IPv6 does not support broadcast address as it is in IPv4, this functionality was replaced by some IPv6 multicast addresses. The specific use of IPv6 addresses based on RFC 3513 [6] is shown in table 1.1. The IPv6 addressing architecture which was initially explained in RFC 3513, now obsolete, is now specified in [9], [4].

Table 1.1: Specific Use of IPv6 [8]

\begin{tabular}{|l|l|l|}
\hline Address type & Binary prefix & IPv6 notation \\
\hline Unspecified & $00 \ldots 0(128$ bits $)$ & $:: / 128$ \\
\hline Loopback & $00 \ldots 1(128$ bits $)$ & $:: 1 / 128$ \\
\hline Multicast & 11111111 & FF00::/8 \\
\hline Link-local unicast & 1111111010 & FE80::/10 \\
\hline Site-local unicast & 1111111011 & FEC0::/10 \\
\hline Global unicast & Everything else & Everything else \\
\hline
\end{tabular}

\subsection{Succinct Discussion of IPv6 Multicast}

IPv6 multicast addresses which was defined in IP Version 6 Addressing Architecture [9]. Multicast is triggered by the receivers' interest. Multicast group contain arbitrary group of receivers that shows interest in receiving a particular multicast datagram. The members can be located anywhere on the Internet or in any private network without any geographical constrain. Receivers that wish to receive multicast data streamed to a particular group have to join the group by sending a Multicast Listeners Discovery (MLD) message to the router they are connected to. Routers use the MLD protocol to learn whether members of a group are present on their directly attached subnets. Hosts join multicast groups by sending MLD report messages [4].

A multicast group address is selected for the members in a multicast group. This group address is use as the destination address by the sender of a multicast datagram to reach all members who have joined the group or shown interest in receiving the datagram.

Membership in a multicast group is dynamic; hosts can join and 
leave at will. In IPv6, multicast address is an identifier for a set of interfaces that typically belong to different nodes and prefixed by ff00::/8 (1111 1111). A packet destined to a multicast address is delivered to all the interfaces identified by this address using best-effort reliability (there is no datagram safety guarantee) [1]. Figure 1.2 shows the format of the IPv6 multicast address.

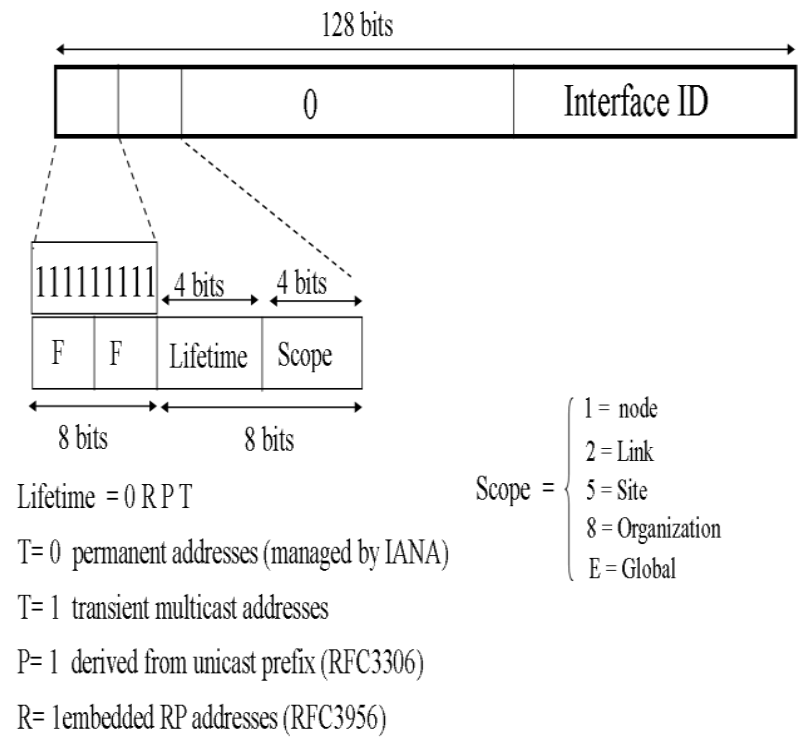

Figure 1.2: IPv6 Multicast Address Format [9]

\subsection{Concept of Virtualization}

In computing, virtualization means creating a virtual (rather than actual) version of something. These include virtual network resources, virtual storage devices, virtual operating system and virtual hardware platform.

Normally, every physical computer has one instance of the operating system which supports one or more application programs; a single physical computer runs software that abstracts the physical computer's resources so that they may be shared between multiple "virtual computers" in a virtualization environment [7]. Each virtual computer may be running a different operating system from all of the other virtual machines on the physical machine. A crash or other program error on any of the virtual machines leaves all of the other virtual machines unaffected [5].

In hardware virtualization, virtualization takes place on the host (physical computer), and the guest which is the machine sitting on the host, is the virtual machine. Host and guest are used to differentiate the program set that runs on the physical machine from that which runs on the virtual machine. With the help of a Hypervisor/Virtual Machine Manager virtual machines can be created (Figure 1.3).

Virtualization can be full, partial, or Para virtualization, these depend on the hardware environment is simulated or whether the guest OS is modified or not.

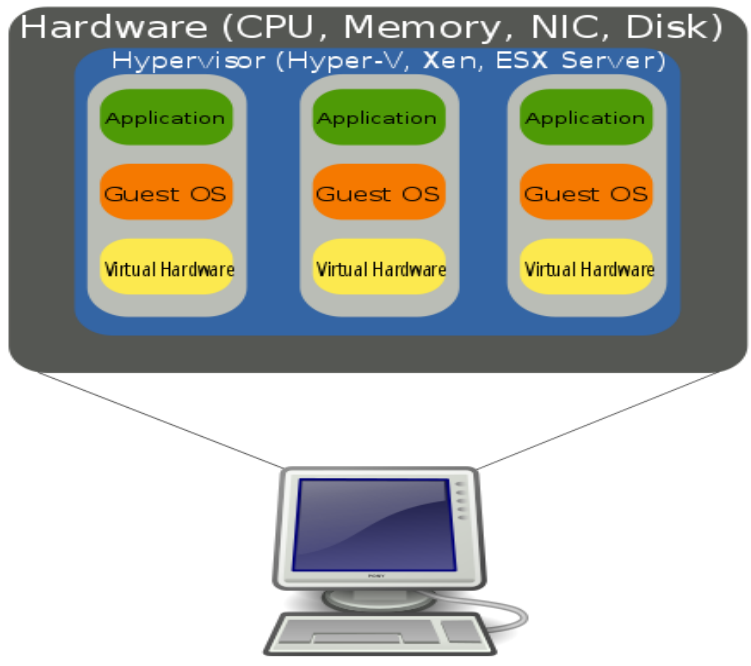

Figure 1.3 Logical Diagram of Full Virtualization [12]

\section{EXPERIMENTAL SETUP}

Graphical Network Simulator 3 was used to configure the network. Microsoft Hyper-V was used as the hypervisor on which the six virtual machines (hosts) reside and a host system running Windows 10 Professional (figure 2.1).

\subsection{System Architecture}

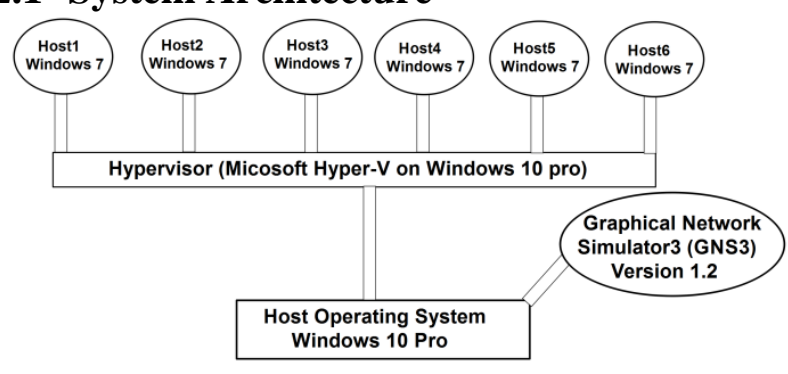

Figure 2.1: System Architecture

\subsection{IPv6 Interface Design}

Figure 2.2 is a virtual network design in GNS3 (Graphical Network Simulator 3). There are three 7200 series router and six hosts that participated in the multicast session. IPv6 unicastrouting, IPv6 multicast-routing, IPv6 Open Shortest Path First (OSPFv3) were all enable on each of the router. Each of the interfaces in the router is configured with IPv6 global unicast address and Protocol Independent Multicast Sparse Mode (PIMSM) enabled. Router 2 loopback0 interface was configured as the Rendezvous point (RP) address. Each of the guest operating system (Arbitrarily named Host1 to Host6) is connected to the Microsoft Hyper- $\mathrm{V}$ adapters on host operating system (figure 2.1) via the cloud and runs JPERF (A Java frontend of Iperf, an application for generating multicast traffics).

The network at this point is routable, that is, from Host1 you can reach all other Hosts and vice versa. 


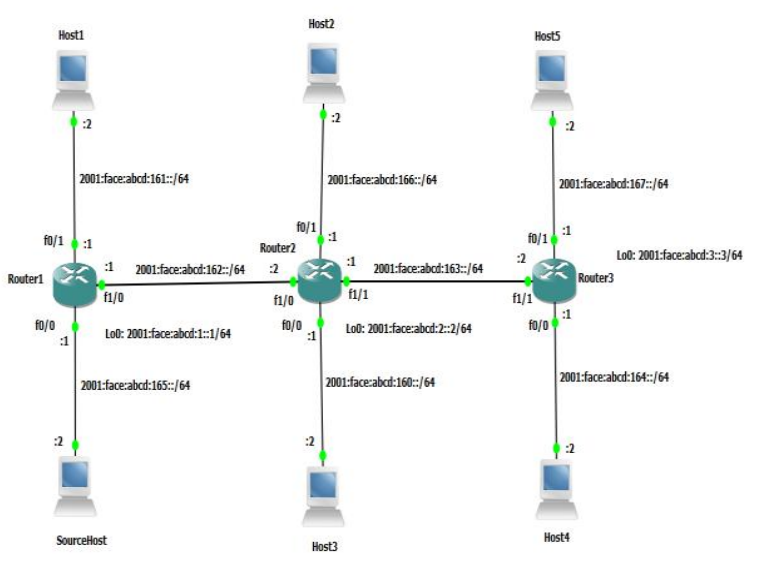

Figure 2.2 IPv6 network interface design

\subsection{Setting Up IPv6 Multicast Routing}

Two testing durations were conducted; three ten minutes runs and three one hour runs.

In the first setup, all the five hosts joined one multicast group.

a. SourceHost was chosen arbitrary (not configured) as the source of the multicast traffic.

b. Host1, Host2, Host3, Host4 and Host5 joined the multicast group ff7e:240:2001:face:abcd:2:0:2

c. SourceHost send packets to this multicast address ff7e:240:2001:face:abcd:2:0:2.

d. Figure 2.3 shows one of the listeners waiting for packets

e. Figure 2.4 shows the responses from the five listeners.

f. All other settings for JPERF take the default values.

g. Table 3.1 shows the average jitters of the five hosts that received a multicast datagram for ten minutes and one hour running and figure 3.1 shows the graph.

In the second scenario,

a. SourceHost and Host1 were chosen as the sources of the multicast traffics.

b. Host 2 and Host 4 joined the multicast group ff7e:240:2001:face:abcd:2:0:3

c. Host 3 and Host5 joined the multicast group ff7e:240:2001:face:abcd:2:0:5

d. SourceHost send packets to this multicast address ff7e:240:2001:face:abcd:2:0:3

Host1 send packets to this multicast address ff7e:240:2001:face:abcd:2:0:5 simultaneously.

e. Table 3.2 shows the jitters for the groups ten minutes and 1 hour streaming.

f. Figure 3.2 shows the jitters' graph for the groups ten minutes and 1 hour streaming.

g. All other settings for JPERF take the default values.

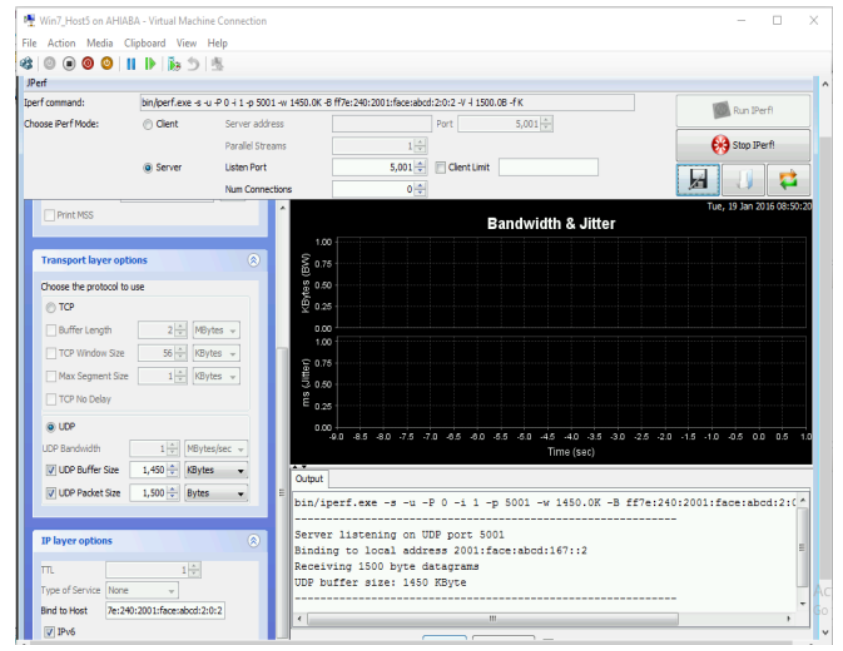

Figure 2.3: One of the five hosts waiting to receive a multicast streaming

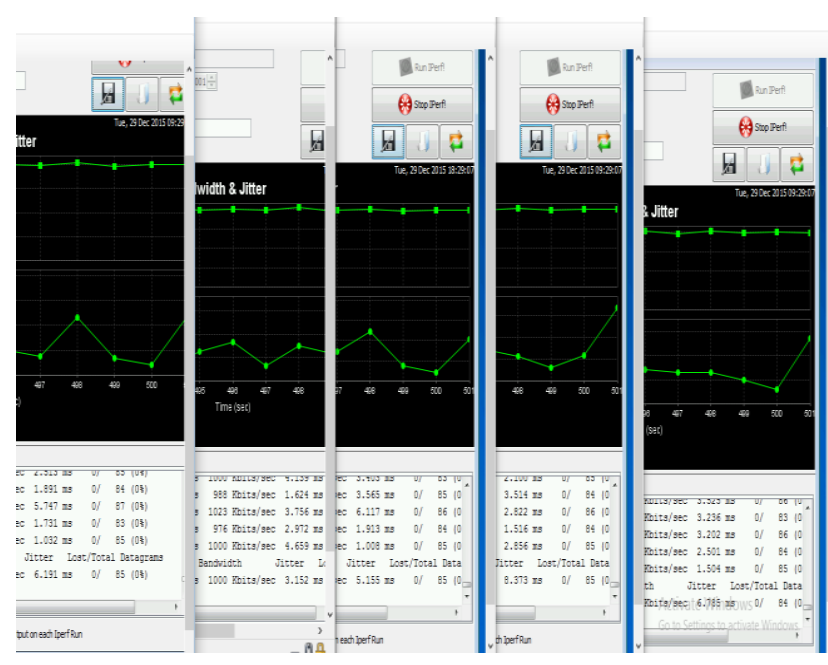

Figure 2.4: All the five hosts receiving a multicast streaming

3. RESULT ANALYSIS

\subsection{Throughput and Latency}

Table 3.1 shows stable jitters among the five hosts that participated in a single multicast stream for both the ten minutes and one hour running. The same stable jitters was noticed when streaming two different multicast datagram simultaneously with two hosts each, participating in the streams for ten minutes and the one run (Table 3.2). Figure 3.1 and figure 3.2 are the graphs for table 3.1 and table 3.2 respectively. Therefore, there was no significant increase in latencies of the receiving hosts as multicast group increase from one to two.

From the result obtained from all the average 10 minutes and average 1 hour runs, the throughput was $100 \%$ and the jitters range from 0 to $5.734 \mathrm{~ms}$ 
International Journal of Applied Information Systems (IJAIS) - ISSN : 2249-0868

Foundation of Computer Science FCS, New York, USA

Volume 11 - No. 3, August 2016 - www.ijais.org

Table 3.1: Test result for five listeners received ten minutes and1hour streams

\begin{tabular}{|c|c|c|c|c|c|}
\hline & Jitters & eiving $\mathrm{l}$ & & & \\
\hline Sender (Source-Host) & Host1 & Host2 & Host3 & Host4 & Host5 \\
\hline 10 min Run & 3.404 & 1.400 & 1.257 & 1.625 & 1.860 \\
\hline $10 \min 2^{\text {nd }}$ Run & 1.980 & 2.532 & 3.448 & 2.563 & 3.269 \\
\hline $10 \min 3^{\text {rd }}$ Run & 1.434 & 2.950 & 1.814 & 1.348 & 1.425 \\
\hline Average of the three $10 \mathrm{~min}$ Run & 2.272 & 2.294 & 2.173 & 1.845 & 2.184 \\
\hline 1hr Run & 1.063 & 1.117 & 1.487 & 1.254 & 0.005 \\
\hline $1 \mathrm{hr} 2^{\text {nd }}$ Run & 5.734 & 4.601 & 2.754 & 2.911 & 1.766 \\
\hline $1 \mathrm{hr} 3^{\text {rd }}$ Run & 0.638 & 1.022 & 1.069 & 0.931 & 0.329 \\
\hline Average of the three $1 \mathrm{hr}$ Run & 2.478 & 2.246 & 1.77 & 1.698 & 0.7 \\
\hline
\end{tabular}

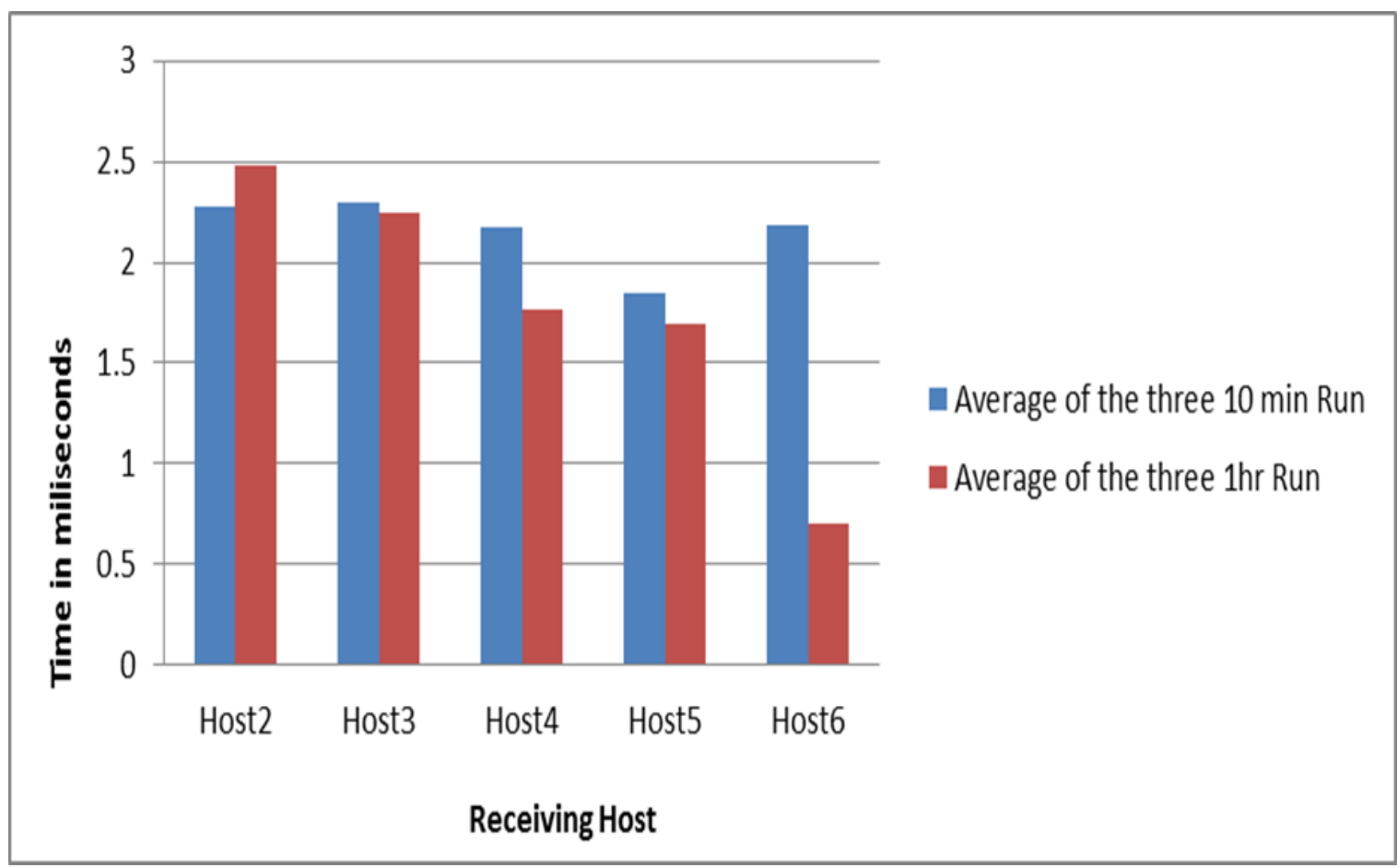

Figure 3.1: Jitter graph for five listeners received ten minutes and1hour streams 
Table 3.2: Test result for running two IPv6 groups simultaneously (ten minutes and1hour streams)

\begin{tabular}{|c|c|c|c|c|}
\hline \multirow[b]{3}{*}{ Durations } & \multicolumn{4}{|c|}{ Jitters of Receiving hosts } \\
\hline & \multicolumn{2}{|c|}{$\begin{array}{l}\text { Source-Host multicast to } \\
\text { ff } 7 \text { e: } 240: 2001: \text { face: abcd }: 2: 0: 3\end{array}$} & \multicolumn{2}{|c|}{$\begin{array}{l}\text { Host1 multicast } \\
\text { ff7e: } 240: 2001: \text { face: abcd }: 2: 0: 5\end{array}$} \\
\hline & Host2 & Host4 & Host3 & Host5 \\
\hline $10 \mathrm{~min}$ Run & 0.429 & 0.694 & 1.853 & 2.107 \\
\hline $10 \min 2^{\text {nd }}$ Run & 1.061 & 2.621 & 1.875 & 1.491 \\
\hline $10 \mathrm{~min} 3 \mathrm{rd}$ Run & 0.539 & 1.594 & 2.004 & 2.027 \\
\hline $\begin{array}{l}\text { Average of the three } 10 \\
\text { min Run }\end{array}$ & 0.676 & 1.636 & 1.91 & 1.875 \\
\hline 1hr Run & 1.979 & 1.694 & 3.727 & 1.971 \\
\hline 1hr $2^{\text {nd }}$ Run & 3.747 & 1.982 & 3.278 & 4.751 \\
\hline 1hr 3rd Run & 0.229 & 1.594 & 3.003 & 2.107 \\
\hline $\begin{array}{l}\text { Average of the three } 1 \mathrm{hr} \\
\text { Run }\end{array}$ & 1.985 & $\mathbf{1 . 7 5 6}$ & 3.336 & 2.943 \\
\hline
\end{tabular}

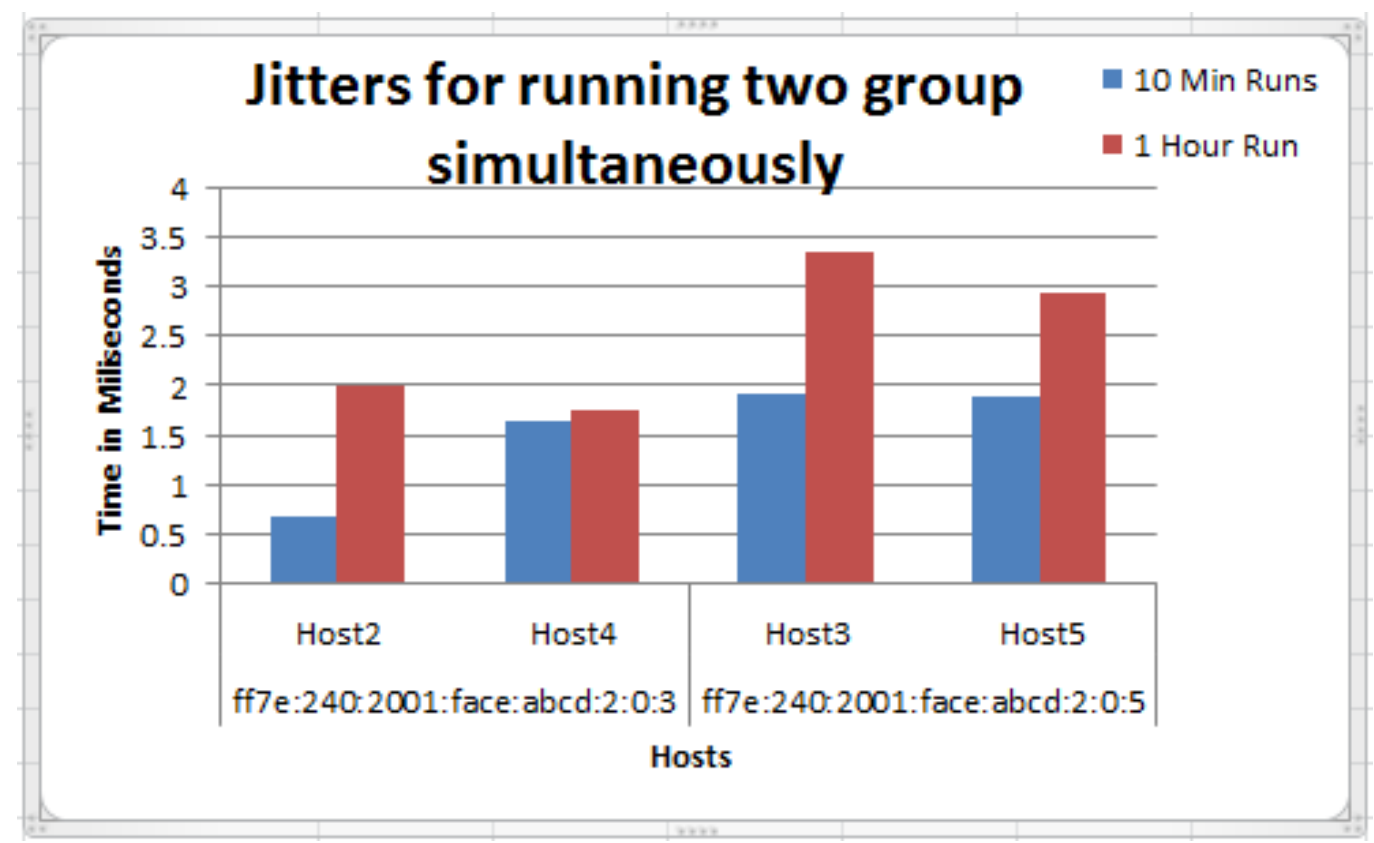

Figure 3.2: Jitter graph for running two IPv6 multicast groups simultaneously (ten minutes and1hour streams)

\subsection{Data Loss}

There was no datagram lost in all the running scenarios. The

zero datagram loss resulted in the $100 \%$ throughput achieved (figure 3.3). 


\begin{tabular}{|c|c|c|c|c|c|c|c|c|c|c|c|}
\hline ID] & Interval & \multicolumn{2}{|c|}{ Transfer } & \multicolumn{2}{|c|}{ Bandwidth } & \multicolumn{2}{|c|}{ Jitter } & \multicolumn{2}{|c|}{ Lost/Total } & \multicolumn{2}{|c|}{ Datagrams } \\
\hline [132] & $3580.0-3581.0$ & $\sec$ & 122 & RBytes & 122 & KBytes/sec & 3.734 & $\mathrm{~ms}$ & $0 /$ & 85 & (08) \\
\hline [132] & $3581.0-3582.0$ & $\sec$ & 122 & RBytes & 122 & KBytes/sec & 3.780 & $\mathrm{~ms}$ & $0 /$ & 85 & (08) \\
\hline [132] & $3582.0-3583.0$ & $\sec$ & 121 & kBytes & 121 & RBytes/sec & 2.116 & $\mathrm{~ms}$ & $0 /$ & 84 & (08) \\
\hline [132] & $3583.0-3584.0$ & sec & 119 & RBytes & 119 & RBytes/sec & 1.282 & $\mathrm{~ms}$ & $0 /$ & 83 & (08) \\
\hline [132] & $3584.0-3585.0$ & $\sec$ & 122 & kBytes & 122 & KBytes/sec & 0.544 & $\mathrm{~ms}$ & $0 /$ & 85 & (08) \\
\hline [132] & $3585.0-3586.0$ & $\sec$ & 121 & RBytes & 121 & RBytes/sec & 2.459 & $\mathrm{~ms}$ & $0 /$ & 84 & (08) \\
\hline [132] & $3586.0-3587.0$ & $\sec$ & 121 & RBytes & 121 & RBytes/sec & 0.547 & $\mathrm{~ms}$ & $0 /$ & 84 & (08) \\
\hline [132] & $3587.0-3588.0$ & $\sec$ & 121 & kBytes & 121 & RBytes/sec & 4.606 & $\mathrm{~ms}$ & $0 /$ & 84 & (08) \\
\hline [132] & $3588.0-3589.0$ & sec & 122 & RBytes & 122 & KBytes/sec & 2.296 & $\mathrm{~ms}$ & $0 /$ & 85 & (08) \\
\hline [132] & $3589.0-3590.0$ & $\sec$ & 119 & RBytes & 119 & KBytes/sec & 6.038 & $\mathrm{~ms}$ & $0 /$ & 83 & (O\&) \\
\hline [132] & $3590.0-3591.0$ & $\sec$ & 119 & RBytes & 119 & RBytes/sec & 1.150 & $\mathrm{~ms}$ & $0 /$ & 83 & (08) \\
\hline [132] & $3591.0-3592.0$ & $\sec$ & 121 & RBytes & 121 & KBytes/sec & 1.871 & $\mathrm{~ms}$ & $0 /$ & 84 & (08) \\
\hline [132] & $3592.0-3593.0$ & $\sec$ & 121 & RBytes & 121 & RBytes/sec & 1.245 & $\mathrm{~ms}$ & $0 /$ & 84 & (08) \\
\hline [132] & $3593.0-3594.0$ & $\sec$ & 122 & RBytes & 122 & KBytes/sec & 0.641 & $\mathrm{~ms}$ & $0 /$ & 85 & (O\&) \\
\hline [132] & $3594.0-3595.0$ & $\sec$ & 121 & RBytes & 121 & RBytes/sec & 0.972 & $\mathrm{~ms}$ & $0 /$ & 84 & (O8) \\
\hline [132] & $3595.0-3596.0$ & $\sec$ & 121 & RBytes & 121 & KBytes/sec & 5.047 & $\mathrm{~ms}$ & $0 /$ & 84 & (08) \\
\hline [132] & $3596.0-3597.0$ & $\sec$ & 122 & RBytes & 122 & RBytes/sec & 1.280 & $\mathrm{~ms}$ & $0 /$ & 85 & (O\&) \\
\hline [132] & $3597.0-3598.0$ & $\sec$ & 119 & RBytes & 119 & RBytes/sec & 3.134 & $\mathrm{~ms}$ & $0 /$ & 83 & (08) \\
\hline [132] & $3598.0-3599.0$ & $\sec$ & 122 & RBytes & 122 & RBytes/sec & 6.967 & $\mathrm{~ms}$ & $0 /$ & 85 & (O\&) \\
\hline [132] & $3599.0-3600.0$ & $\sec$ & 121 & KBytes & 121 & KBytes/sec & 0.588 & $\mathrm{~ms}$ & $0 /$ & 84 & (08) \\
\hline [ ID] & Interval & Tre & sfer & Ban & vidth & Jitt & $x$ & & tal & Datag & rams \\
\hline [132] & $0.0-3600.0 \mathrm{se}$ & & 4129 & KBytes & 121 & KByte & 0.517 & $m$ & & 30241 & $(0 \&)$ \\
\hline
\end{tabular}

Figure 3.3: Screen Shot of a Host with No Data Loss

\subsection{Protocol Overheads}

The following are the protocols over heads noticed (figure 3.4 deduced from the Wire shark captures).

a. PIM-SM was used as the multicast routing protocol. The protocol did not produce much of an overhead The PIMv2 Hello messages were sent out at irregular intervals.

b. OSPF was used as the unicast routing protocol. It sends updates and acknowledgement messages to all routers multicast address ff02::5 at a regular interval $30 \mathrm{~ms}$.

c. ICMPv6 neighbour solicitation and neighbour advertisement messages were also noticed.

d. IPv6 fragmented packet offsets messages are sent the embedded rendezvous point address ff7e:240:2001:face:abcd:2:0:2 about every $0.020767 \mathrm{~ms}$ or less.

\begin{tabular}{|c|c|c|c|c|}
\hline No. & Time & Source & Destination & Protocol \\
\hline 6353 & 39.226133000 & 2001:face:abcd:160:7d35:77a8:c777:c3a6 & ff7e:240:2001:face:abcd:2:0:2 & IPV6 \\
\hline 6354 & 39.226133000 & 2001:face:abcd:160:7d35:77a8:c777:c3a6 & ff7e: $240: 2001:$ face $:$ abcd $: 2: 0: 2$ & UDP \\
\hline 6355 & 39.226133000 & 2001:face:abcd:160:7d35:77a8:c777:c3a6 & ff7e:240:2001:face:abcd:2:0:2 & IPV6 \\
\hline 6356 & 39.226133000 & $2001:$ face: abcd:160:7d35:77a8:c777:c3a6 & ff7e: $240: 2001:$ face $:$ abcd: $: 2: 0: 2$ & UDP \\
\hline 6357 & 39.246900000 & 2001:face:abcd:160:7d35:77a8:c777:c3a6 & ff7e:240:2001:face:abcd:2:0:2 & IPV 6 \\
\hline 6358 & 39.247768000 & $2001:$ face $:$ abcd: $160: 7 \mathrm{~d} 35: 77$ a $8: c 777: c 3 a 6$ & ff7e: $240: 2001:$ face $:$ abcd: $: 2: 0: 2$ & UDP \\
\hline 6359 & 39.263406000 & fe80::c803:3bff:fe94:8 & ffo $02:: d$ & PIMV2 \\
\hline 6360 & 39.263406000 & 2001:face:abcd:160:7d35:77a8:c777:c3a6 & ff7e:240:2001:face:abcd:2:0:2 & IPV 6 \\
\hline 6361 & 39.263406000 & 2001:face:abcd:160:7d35:77a8:c777:c3a6 & ff7e:240:2001:face:abcd:2:0:2 & UDP \\
\hline 6362 & 39.279049000 & 2001:face:abcd:160:7d35:77a8:c777:c3a6 & ff7e:240:2001:face:abcd:2:0:2 & IPV6 \\
\hline 6363 & 39.279049000 & 2001:face:abcd:160:7d35:77a8:c777:c3a6 & ff7e:240:2001:face:abcd:2:0:2 & UDP \\
\hline 6364 & 39.294665000 & 2001:face:abcd:160:7d35:77a8:c777:c3a6 & ff7e:240:2001:face:abcd:2:0:2 & IPV 6 \\
\hline 6365 & 39.294665000 & 2001:face:abcd:160:7d35:77a8:c777:c3a6 & ff7e:240:2001:face:abcd:2:0:2 & UDP \\
\hline 6366 & 39.310282000 & 2001:face:abcd:160:7d35:77a8:c777:c3a6 & ff7e:240:2001:face:abcd:2:0:2 & IPV6 \\
\hline 6367 & 39.310282000 & $2001:$ face: abcd:160:7d35:77a8:c777:c3a6 & ff7e: $240: 2001:$ face $:$ abcd: $: 2: 0: 2$ & UDP \\
\hline 6368 & 39.325906000 & fe80: :c803:3bff:fe94:8 & ffo $02:$ : & PIMV2 \\
\hline 6369 & 39.325906000 & fe $80:: c 801: 36 f f: f e 24: 8$ & fe $80:: c 802: 38 f f:$ fef $4: 6$ & ICMPV6 \\
\hline 6370 & 39.325906000 & 2001:face:abcd:160:7d35:77a8:c777:c3a6 & ff7e:240:2001:face:abcd:2:0:2 & IPV6 \\
\hline 6371 & 39.325906000 & $2001:$ face $:$ abcd: $160: 7 d 35: 77$ a $8: c 777: c 3 a 6$ & ff7e: $240: 2001:$ face $:$ abcd $: 2: 0: 2$ & UDP \\
\hline 6372 & 39.342043000 & 2001:face:abcd:160:7d35:77a8:c777:c3a6 & ff7e:240:2001:face:abcd:2:0:2 & IPV 6 \\
\hline 6373 & 39.342043000 & $2001:$ face: abcd:160:7d35:77a8:c777:c3a6 & ff7e: $240: 2001:$ face $:$ abcd: $: 2: 0: 2$ & UDP \\
\hline 6374 & 39.348041000 & 2001:face:abcd:160:7d35:77a8:c777:c3a6 & ff7e:240:2001:face:abcd:2:0:2 & IPV6 \\
\hline 6375 & 39.348041000 & $2001:$ face: abcd:160:7d35:77a8:c777:c3a6 & ff7e: $240: 2001:$ face $:$ abcd $: 2: 0: 2$ & UDP \\
\hline 6376 & 39.379337000 & 2001:face:abcd:160:7d35:77a8:c777:c3a6 & ff7e:240:2001:face:abcd:2:0:2 & IPV6 \\
\hline 6377 & 39.379337000 & 2001:face:abcd:160:7d35:77a8:c777:c3a6 & ff7e:240:2001:face:abcd:2:0:2 & UDP \\
\hline 6378 & 39.379337000 & fe $80:: c 802: 38 f f: f e f 4: 6$ & fe80: :c801:36ff:fe24:8 & ICMPV 6 \\
\hline 6379 & 39.379337000 & 2001:face:abcd:160:7d35:77a8:c777:c3a6 & ff7e:240:2001:face:abcd: $2: 0: 2$ & IPV6 \\
\hline 6380 & 39.379337000 & $2001:$ face: abcd: $160: 7 \mathrm{~d} 35: 77 \mathrm{a} 8: c 777: c 3 a 6$ & ff7e: $240: 2001:$ face $:$ abcd: $: 2: 0: 2$ & UDP \\
\hline 6381 & 39.394975000 & 2001:face:abcd:160:7d35:77a8:c777:c3a6 & ff7e:240:2001:face:abcd:2:0:2 & IPV6 \\
\hline 6382 & 39.394975000 & $2001:$ face $:$ abcd: $160: 7 d 35: 77 a 8: c 777: c 3 a 6$ & ff7e:240:2001:face:abcd:2:0:2 & UDP \\
\hline 6383 & 39.410554000 & fe80::c802:38ff:fef $4: 8$ & ffo2:: 5 & OSPF \\
\hline 6384 & 39.410554000 & fe80::c802:38ff:fef $4: 6$ & ffo2::5 & OSPF \\
\hline
\end{tabular}

Figure 3.4: Wire shark data capture to show protocols over heads 


\subsection{Tools Used}

a. The virtual machines run Windows 7 pro edition

b. Microsoft Hyper-V (Hypervisor)

c. Graphical Network Simulator 3 (Configured the logic of the virtual network with 3 routers 7200 series)

d. Wire Shark (Data Captures)

e. JPERF (Traffic Generator)

f. Host system (Intel Core i5, Windows 10 Pro, RAM 6GB, CPU 2.4GHz, Hard Disk $750 \mathrm{~GB}$ )

\section{CONCLUSION}

From the experiment, IPv6 multicast routing over a virtual network has $100 \%$ throughput, the jitter (variations in latency) varies among the hosts in all the running scenarios, but low and stabled jitters were noticed as the running duration increases and the number multicast stream increased from one to running two simultaneously. No data loss was noticed in all the run. IPv6 multicast routing was successfully demonstrated among six participating hosts on a virtual local area network. However, this paper has the following limitations:
a. The experiment was carried out in a virtual lab not a real lab.
b. The datagram tested are not real application data. depend on resource of the host system. Host with better resources may better performance.
c. The resources available for the virtual machines

\section{REFERENCES}

[1] Cisco. (2001). Overview of IP Multicast. Retrieved March 12, 2013, from http://www.cisco.com/en/US/tech/tk828/technologies_w hite_paper09186a0080092942.shtml

[2] Daniel, M (2001). Voice Over IPv6: Architecture for Next Generation VoIP Networks. Linacre House, Jordan Hill. Oxford Ox2 8DP, USA, pg 251-291.
[3] Deering, S., \& Hinden, R. (1998). RFC 2460 Internet Protocol Version 6 (IPv6) Specification. Retrieved on March 17, 2014, from https://www.ietf.org/rfc/rfc2460.txt

[4] Deering, S., \& Hinden, R. (2006): IP Version 6 Addressing Architecture. Retrieved 17 March, 2014 from http://tools.ietf.org/html//rfc4291

[5] IBM (2007): Virtualization in Education. White Paper Retrieved 27 May 2014 from http://www07.ibm.com/solutions/in/education/download/Virtualizati on $\% 20$ in $\% 20$ Education.pdf

[6] Network Working Group. (1998). RFC 2460 Internet Protocol Version 6 (IPv6) Specification. Retrieved on March 17, 2014, from https://www.ietf.org/rfc/rfc2460.txt

[7] Osero, B. O. \& Mwathi, D. G. (2014). Implementing Security on virtualized network storage environment. International Journal of Education and Research, 2(4), 110

[8] RFC 2460. IPv6 Header. Retrieved on February 12, 2014 from https://en.wikipedia.org/wiki/IPv6_packet

[9] RFC 4291. IP Version 6 Addressing Architecture . Retrieved on 12 February, 2014 from http://tools.ietf.org/html/rfc4291

[10] Webber, J. (2013). IPv6 Test Laboratory. Retrieved on March

17, 2014, from https://www.hpc.mil/images/hpcdocs/ipv 6/masterthesis_johannes_weber_ipv6securitytestlaborato ry.pdf

[11] Wikipedia: IPv6 Packet Header. Retrieved on 17 March, 2014, from https://en.wikipedia.org/wiki/IPv6_packet

[12] Wikipedia. Logical Diagram of Full Virtualization. Retrieved on 18 January, 2016 from http://isa.unomaha.edu/wpcontent/uploads/2012/08/Virtualization.pdf 